\title{
Turbulent transport across shear layers in magnetically confined plasmas
}

\author{
B. Nold ${ }^{1}$, P. Manz ${ }^{2,3}$, T.T. Ribeiro ${ }^{3}$, G. Fuchert ${ }^{4}$, G. Birkenmeier ${ }^{2,3}$, \\ H.W. Müller ${ }^{3}$, M. Ramisch ${ }^{1}$, B.D. Scott ${ }^{3}$ and U. Stroth ${ }^{3,2}$ \\ ${ }^{1}$ Institut für Grenzflächenverfahrenstechnik und Plasmatechnologie, \\ Universität Stuttgart, D-70569 Stuttgart, Germany \\ 2 Physik-Department E28, Technische Universität München, \\ James-Franck-Str.1, D-85748 Garching, Germany \\ 3 Max-Planck-Institut für Plasmaphysik, Boltzmannstr.2 ,D-85748 Garching, Germany \\ 4 IJL, Université de Lorraine, CNRS (UMR 7198), BP 40239, Vandoeuvre-lès-Nancy, France
}

(Dated: September 15, 2014)

\begin{abstract}
Shear layers modify the turbulence in diverse ways and do not only suppress it. A spatialtemporal investigation of gyrofluid simulations in comparison with experiments allows to identify further details of the transport process across shear layers. Blobs in and outside a shear layer merge, thereby exchange particles and heat and subsequently break up. Via this mechanism particles and heat are transported radially across shear layers. Turbulence spreading is the immanent mechanism behind this process.
\end{abstract}

PACS numbers:

\section{INTRODUCTION}

A large contribution to the transport of particles and energy in the scrape-off layer (SOL) of magnetically confined fusion devices is due to filamentary structures called blobs [1]. Their potential to damage the first vessel walls depends on their density and temperature which are determined on one hand by their generation process close to the hot and dense confined plasma and on the other hand by their propagation process. In this article blob refers to a coherent structure with high density with respect to the surrounding fluctuation level. It is widely accepted that poloidal shear flows increase the particle and energy confinement in fusion devices [2-6]. Recently, the extraordinary role of shear layers for blob generation has been identified [7-12]. A picture of blob ejection depending on the shearing rate has been presented in Ref. [13]: Turbulent structures are expected to be radially extended and are more like streamers than isotropic blobs, if shear flows are absent. These structures are explained by curvaturedriven interchange modes, which are supposed to arise close to the maximum normalized pressure gradient. In weak shear flow regimes, streamers generated inside the confined plasma are expected to break up into blobs at the shear layer [13]. In strong shear flow regimes, streamers are expected to be strongly suppressed and blob production should be low [13]. This mechanism of blob ejection applies for radially elongated structures, e.g. streamers, that are generated inside the confined plasma and subject to eddy breaking. However, poloidally elongated structures are subject to eddy tilting only [14]. Even at rather high shearing rates achieved in biasing induced confinement transitions eddies do not break up $[15,16]$. To the best of our knowledge, the only evidence for eddy breaking can be found in Ref. [14]. There, eddy breaking occurs only in the strong shear regime, below a critical threshold the eddies are only tilted [14] and subject to vortex-thinning [17-19].
A closer look to the dynamics at a shear layer in ASDEX Upgrade reveals two peaks in the cross-correlation of two poloidally separated probes indicating blobs poloidally propagating into opposite directions [20]. Also in ALCATOR C-Mod a crossover of two opposite poloidal velocities at the shear layer has been shown [21] and in TJ-II two opposite poloidal velocities are observed at a shear layer in the confined region [22]. How these poloidal velocities are related to the radial transport is studied here. It has been reported that drift waves inside the shear layer are correlated with blobs outside of it $[9,23,24]$. Drift waves preferentially propagate in the poloidal direction and not in the radial one across the shear layer as interchange modes do. In this presentation, experimental data which in the present detail can only be obtained in a low temperature plasma device as the one in the TJ-K stellerator [25], and gyrofluid simulations carried out with the GEMR code [26] are studied to develop a drift-wave based model for blob ejection consistent with the recent observations in the high temperature plasmas in ASDEX Upgrade, Alcator C-Mod and TJ-II [20-22].

\section{TJ-K EXPERIMENTS}

The dynamics of blob ejection was measured in lowtemperature plasmas of the stellarator experiment TJ-K. The major and minor plasma radii of TJ-K are $R_{0}=0.6$ $\mathrm{m}$ and $a=0.1 \mathrm{~m}$, respectively. Long time series can be measured as they are necessary for detailed statistical investigations. This is not possible in high-temperature fusion devices and extremely demanding in numerical simulations. The ion temperature of TJ-K plasmas is less than $1 \mathrm{eV}[27]$ and the electron temperature is constant at about $T_{e}=10 \mathrm{eV}$. The working gases were hydrogen and helium. Plasma was generated by microwaves at $2.45 \mathrm{GHz}$ and $1.8 \mathrm{~kW}$ [28]. The line-averaged density 


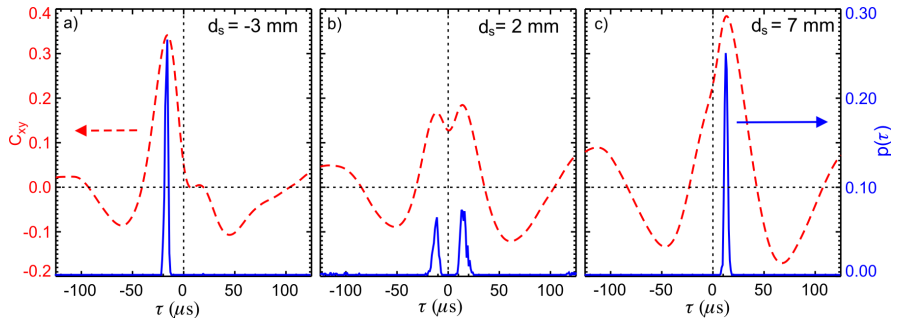

FIG. 1: Cross-correlation of ion-saturation current fluctuations (dashed, red line) and probability density of the crosscorrelation maxima of subwindows with $4 \mathrm{~ms}$ duration (solid, blue line) with varying distances to the LCFS. The poloidal probe distance is $2.5 \mathrm{~cm}$. (TJ-K, \#5090, H, $2.45 \mathrm{GHz}$ )

was about $\bar{n}=10^{17} \mathrm{~m}^{-3}$ and the magnetic field strength was $B=70 \mathrm{mT}$.

The poloidal velocity of turbulent structures in TJ-K seems to change abruptly at a shear layer and not continuously with the $E_{\mathrm{r}} \times B$-drift [9]. The present contribution investigates this shear layer in more detail, taking advantage of the long-lasting and stable plasma discharges in a stellarator. Ion-saturation current fluctuations have been recorded simultaneously with two poloidally separated Langmuir probes. The sampling rate and resolution of the data were $1 \mathrm{MHz}$ and $16 \mathrm{bit}$, respectively. The red dashed lines in Fig. 1 show the cross-correlation function of these signals at three different radial positions in the midplane of an outer port. The correlation functions inside and outside of the last closed flux surface (LCFS) show a clear maximum with negative (Fig. 1a) and positive time delay $\tau$ (Fig. 1c), respectively. This indicates, that turbulent structures in the confined plasma $\left(d_{\mathrm{s}}<0 \mathrm{~mm}\right)$ propagate poloidally in the electron diamagnetic direction (EDD). Structures in the SOL $\left(d_{\mathrm{S}}>0 \mathrm{~mm}\right)$ propagate in the ion diamagnetic direction (IDD). A continuous shear layer is expected in between these two radial measurement positions. The poloidal velocity of radially propagating structures should be reduced in the shear layer before they are accelerated in the opposite direction. Structures with low poloidal velocities should cause large time delays of the correlation maxima. Mainly radially propagating structures should cause small time delays of the correlation maxima. Instead of this expectation, a double peak is observed in the cross-correlation functions close to the LCFS in TJ-K (Fig. 1b). The $512 \mathrm{~ms}$ measurements have been cut into 128 sub-series and separately correlated to check whether a reduced poloidal velocity would show up at least in some of these $4 \mathrm{~ms}$ sub-series. The probability distributions of the corresponding correlation maxima are shown as solid blue lines in Fig. 1 for the same radial positions. Even the short sub-series are dominated by structures propagating either with one or the other velocity in different directions. This finding qualitatively reproduces recent results from different experiments based on different diagnostics [9, 20-22].

Ion-saturation current fluctuations have been mea-

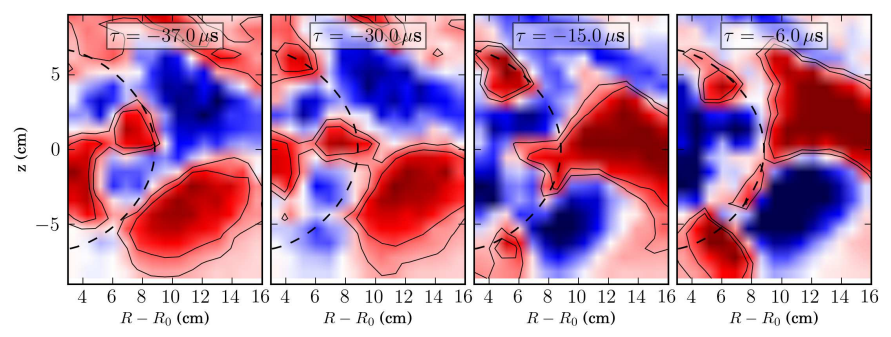

FIG. 2: Poloidal cross section of conditional averaged ionsaturation current fluctuations at different time lags $\tau$. (TJK, \#8211, He, $2.45 \mathrm{GHz}$ )

sured over a whole poloidal cross-section of TJ-K to study the spatio-temporal behavior of turbulent structures. A 2D movable Langmuir probe scanned the plasma at an outer port with a step size of $1 \mathrm{~cm}$ in both directions. A second fixed probe provided a reference signal for conditional averaging [29], where $\tilde{n}_{\mathrm{e}}=2 \sigma$ is used as the trigger condition with $\sigma$ the standard deviation of the signal. Using such a trigger condition, blobs are identified as coherent structures with high density in respect to the surrounding fluctuation level.

Figure 2 represents coherent fluctuations with positive and negative amplitudes by red and blue color with and without contour lines, respectively. The dashed circle marks the LCFS and the center of the plasma is located at $R-R_{0}=4 \mathrm{~cm}$ and $z=0 \mathrm{~cm}$. Inside the LCFS, a driftwave structure propagates clockwise, i.e. in the EDD. In the SOL, a high density structure propagates counterclockwise in the IDD. Both structures are separated in the first frame, touch each other in the second, unify to one radially extended structure in the third and get sheared apart in the last frame. Size and density of the drift-wave structure inside the shear layer have been substantially reduced during this process. This could be explained by radial transport in the unified structure across the shear layer.

\section{GEMR SIMULATION}

Of course, the conditions in TJ-K experiments differ from high temperature plasmas, where measurements with comparable duration and spatial resolution are not feasible. Simulations at ASDEX Upgrade relevant plasma parameters [30] have been carried out to show relevance of the observation for fusion devices. Simulations provide the unique possibility to observe all relevant quantities simultaneously. However, the ejection mechanism of plasma blobs can only be observed in simulations with self-consistently generated instead of seeded blobs. Such a simulation is provided by the three-dimensional gyrofluid turbulence model GEMR [26]. The GEMR model assumes a circular cross-section with toroidal axisymmetry. The coordinate system is aligned with the equilibrium magnetic field. A set of grid points with con- 

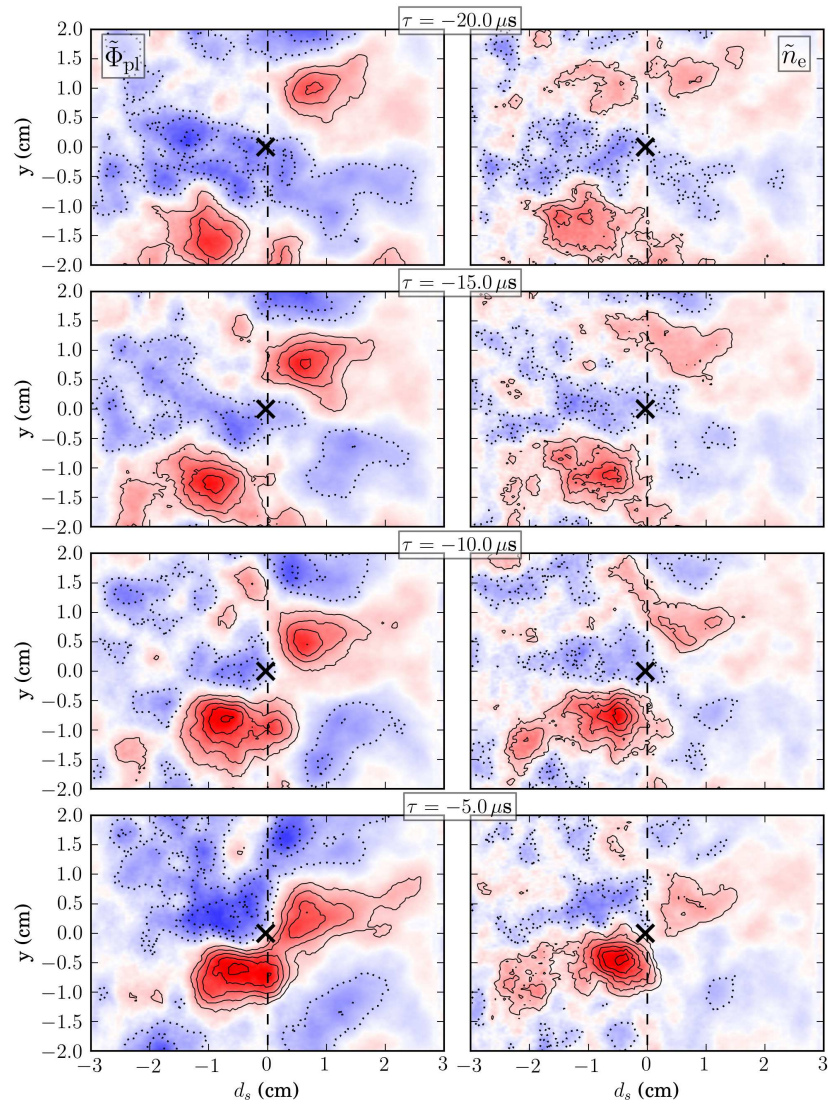

FIG. 3: Conditional averaged turbulent structures in the plasma potential (left-hand side) and density (right-hand side), respectively, in the GEMR simulation at different time lags $\tau$ to the fulfilling of the trigger condition at the position of the black cross. The dashed and solid lines indicate negative and positive values, respectively. The difference between two lines is $1 \cdot 10^{17} \mathrm{~m}^{-3}$ and $1 \mathrm{~V}$, respectively.

stant parallel coordinate represents a drift plane, which is perpendicular to the magnetic field.

The open field lines corresponding to the SOL are implemented by the boundary conditions via a perturbed Debye sheath current [31], such to retain the conducting wall instability [31]. The actual boundary conditions used in the simulation, which include the temperature dynamics, can be found in Ref. [32]. Hence, the GEMR model includes, besides the standard interchange physics in tokamak geometry at the transition from closed to open field lines, also the drift-wave and the conducting wall instability. Although being a $\delta$-f limited code the gradients evolve freely, as required by the strength of fluctuating dynamics in this plasma region. However, the deviation from the preset background values has to be small. Within this framework, experimental and modeling results have already been compared with reasonably good agreement [30, 33-35].

In sum, most of the effects which are believed to play an important role for the plasma blob dynamics are included at least in the near SOL, namely, three-

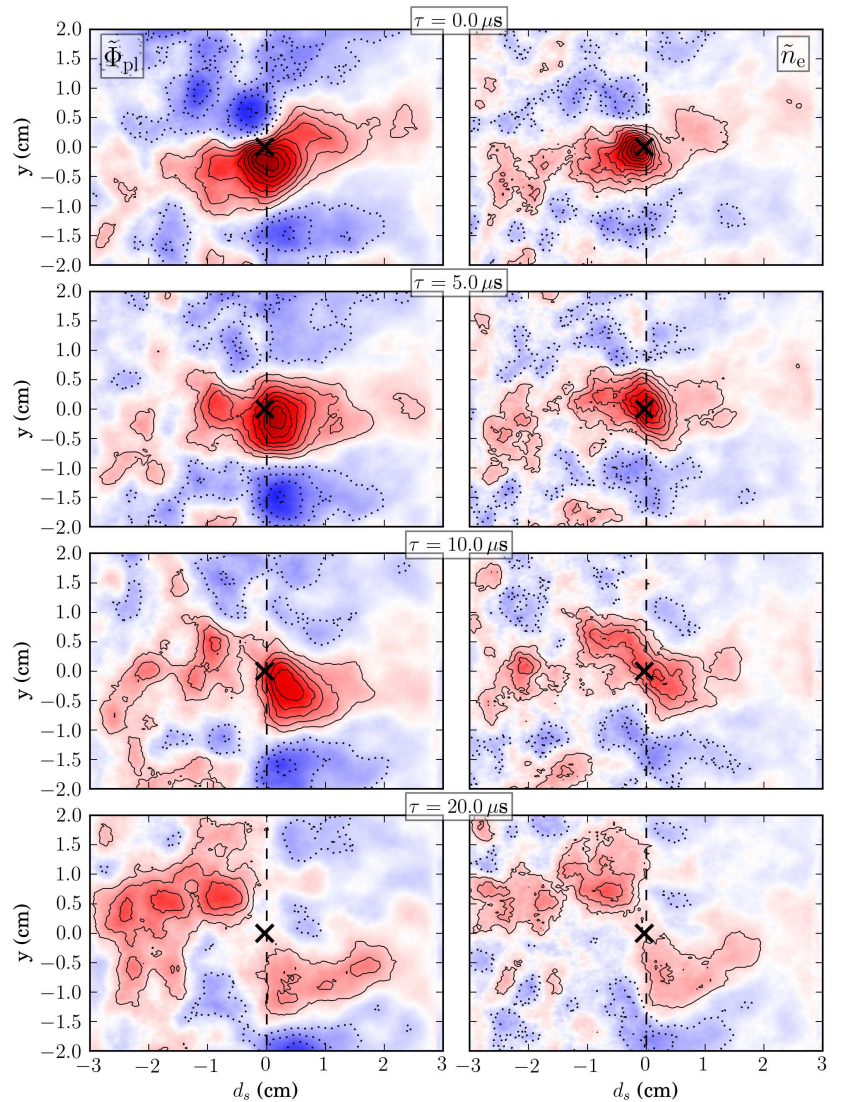

FIG. 4: Continuation of Fig. 3 after fulfilling the trigger condition.

dimensional [36], electromagnetic [37], finite ion temperature or finite Larmor radius [38-40] and shear flow (amongst others the zonal flow and the geodesic acoustic mode) effects $[7,8,11]$. More details on the present simulation parameters can be found in Ref. [30], because the same data is analyzed in the following. Also for the numerical data conditional average is used for the analysis, therefore blobs refer to high coherent density perturbations in respect to the surrounding fluctuation level.

In Ref. [32] it is shown that the turbulence in SOL region is not generated there, but rather in the edge region, which serves as a source. By crossing the LCFS the turbulence changes characteristic to interchange due to changes in the parallel boundary conditions. The phase distribution of density ahead of potential fluctuations increases from small values in the plasma edge (typical for drift-waves) to values closer to $\pi / 2$ in the SOL (indicative for interchange) [32]. The typical edge/SOL transition is found to occur over 10-20 $\rho_{s}$ corresponding to roughly 1 $\mathrm{cm}$ into the SOL which is in accordance to the shear layer width [32]. In the present simulation as in recent observations in ASDEX Upgrade the region around the LCFS is drift-wave dominated [30]. This region is investigated in detail in the present contribution.

Figures 3 and 4 show the conditional average of plasma 
potential (left) and density fluctuations (right) in GEMR at different time lags $\tau$. The analyzed data represents the outer midplane. The reference signal (black cross) to trigger the events was taken from close to the LCFS (dashed vertical line) and the magnetic field is pointing towards the observer. Solid contour lines indicate positive fluctuations (red background) and dashed contour lines negative fluctuations (blue background). The positive potential structures are surrounded by an electric field that causes a clockwise $E \times B$-drift along the equipotential lines. Two such vortices can be seen in the top of Fig. 3. Their positive potentials in the left figures nearly coincide with positive density amplitudes in the right figures. The vortex shown left of the LCFS, i.e. in the confined plasma, propagates in the following frames upward in the EDD. The vortex shown right of the LCFS, i.e. in the SOL, propagates in the following frames downward in the IDD. Both structures touch each other and connect $5 \mu \mathrm{s}$ before the trigger condition $\left(\tilde{n}_{\mathrm{e}}=2 \sigma\right)$ is fulfilled. In the bottom of Fig. 3, the potential structures have united to one single structure, which is extended across the shear layer. This structure depicts the streamline configuration of a vortex merger [41]. Around the two local maxima an equipotential line has developed, called the exchange band [41]. Via the exchange band particles and energy can be transferred from one vortex to the other. In the top of Fig. 4, the density blobs have also united to one single structure. The maximum density is reached in this frame and triggers the conditional averaging $(\tau=0 \mu \mathrm{s})$. Potential and density perturbations are roughly in phase which is a clear feature of drift-wave dynamics. The poloidal gradient of the plasma potential reaches its maximum in the same frame close to the trigger position. A dipole appears in the coherent plasma potential fluctuations inside the LCFS. This dipole advects particles via the $E \times B$-drift radially across the shear layer and LCFS into the SOL. The dipole is not centered above the density perturbation. Although potential and density are still roughly in phase, transport results from the remaining phase shift between density and potential perturbations. The poloidal background flow is continuously active and shears the radially extended structure in the following frames apart. This is similar to the standard break-up of a radial streamer by the action of shear flow. In contrast to this standard picture, the break-up is strongly correlated to the merging process. About $20 \mu \mathrm{s}$ later, the two coherent structures are again separated and propagate in opposite directions.

The observed process describes turbulent transport across a shear layer on a microscopic level. It can be put in an extended context by turbulence spreading, where turbulent structures radially transport energy by merging and breaking up [42]. In general turbulence spreading does not require a sheared background flow. By shrinking the resonance manifold of the nonlinear interactions, the energy cascade is limited to large-scale structures [43], small-scale structures can only couple to the shear flow, where they are absorbed [17]. The resulting effective growth rate is reduced in wavenumber space [43], hence only large-scale structures (the blobs) remain. In general turbulence spreading can be expected to be very important for the far SOL [44], where the mean gradients are found to be flat. Thus, any strong events in that region cannot be generated by the local background gradient itself [44].

\section{DISCUSSION}

Shear layers with two distinct opposite velocities have been observed recently with different diagnostics and in various environments. In the stellarator TJ-K, such a shear layer has been identified with Langmuir probes at the LCFS of a low temperature plasma [9] and explained by a new mechanism in the present contribution. A similar mechanism was observed in the here presented GEMR simulations of a tokamak with high temperature plasma. Two opposed poloidal velocities have been resolved experimentally by Doppler reflectometry in the confined plasma of the TJ-II stellarator [22]. The analysis of kfspectra from gas-puff-imaging revealed two distinct opposed velocities around the LCFS of the tokamak Alcator C-mod [21]. Cross-correlation functions of Langmuir probe measurements in the SOL of the tokamak ASDEX Upgrade revealed a similar finding [20]. All this experimental evidence indicates that shear layers with two opposite directed poloidal velocities might be a general phenomenon. By definition the shear flow exhibits radially different velocities which in a suitable frame of reference appear in opposite directions. The presented mechanism relies on a difference in the poloidal velocity. Hence, it should occur wherever flow shear is present. At the reference position where the flow velocity vanishes also two distinct opposite velocities can be expected.

In high temperature tokamaks, the radial electric field $E_{\mathrm{r}}$ has been found to change its sign outside of the LCFS $[20,21]$. The origin of shear layers in the SOL can be understood considering the divertor geometry. The poloidal magnetic field around the X-point is close to zero. This leads to a steep increase of the distance to the wall along magnetic field lines close to the LCFS. Parallel gradients can reduce the electron temperature in front of the target close to the LCFS much stronger than far outside the LCFS. This leads to a peak in the electron temperature in front of the divertor tiles, which is radially displaced from the strike point of the LCFS [45, 46]. The displacement of the peak temperature in the divertor can lead, via the sheath potential, to the observed $E_{\mathrm{r}} \times B$-shear layer in the SOL [47].

\section{CONCLUSION}

It is commonly assumed that blobs are peeled off from larger structures by the shear flow [13]. In the present contribution a different mechanism of particle transport 
across shear layers has been proposed, which is consistent with the two poloidal velocities of opposite sign previously reported in high temperature fusion experiments [20-22]. Due to the background mean sheared flow, blobs propagate in opposite poloidal directions inside and outside of the shear layer. When a blob from inside of the shear layer meets one outside of it, they connect and merge to one radially extended blob. The merged structures exchange particles and heat, which are transported from the hotter and denser region to the colder and less dense region radially outwards. Later on, the structure breaks up again in the background shear and leaves a blob like structure outside of the shear layer. This behavior is reminiscent of turbulence spreading. The blob generated in the SOL propagates not only poloidally with the background flow, but also radially outward. How this mechanism contributes to the turbulence level in the near SOL quantitatively will be presented in a subsequent study [48].
[1] J. Boedo, D. Gray, P. W. Terry, S. Jachmich, G. R. Tynan, R. W. Conn, and TEXTOR-94 team, Nucl. Fusion 42, 117 (2002).

[2] G. D. Conway, C. Angioni, F. Ryter, P. Sauter, and J. Vicente, Phys. Rev. Lett. 106, 065001 (2011).

[3] L. Schmitz, L. Zeng, T. L. Rhodes, J. C. Hillesheim, E. J. Doyle, R. J. Groebner, W. A. Peebles, K. H. Burrell, and G. Wang, Phys. Rev. Lett. 108, 155002 (2012).

[4] P. Manz, G. S. Xu, B. N. Wan, H. Q. Wang, H. Y. Guo, I. Cziegler, N. Fedorczak, C. Holland, S. H. Müller, S. C. Thakur, et al., Phys. Plasmas 19, 072311 (2012).

[5] G. R. Tynan, M. Xu, P. H. Diamond, J. A. Boedo, I. Cziegler, N. Fedorczak, P. Manz, K. Miki, S. Thakur, L. Schmitz, et al., Nucl. Fusion 53, 073053 (2013).

[6] G. Birkenmeier, M. Ramisch, B. Schmid, and U. Stroth, Phys. Rev. Lett. 110, 145004 (2013).

[7] I. Furno, B. Labit, M. Podesta, A. Fasoli, S. H. Müller, P. Poli, F. M.and Ricci, C. Theiler, S. Brunner, A. Diallo, and J. Graves, Phys. Rev. Lett. 100, 055004 (2008).

[8] G. S. Xu, V. Naulin, W. Fundamenski, C. Hidalgo, J. A. Alonso, C. Silva, B. Gonalves, A. H. Nielsen, J. J. Rasmussen, S. Krasheninnikov, et al., Nuclear Fusion 49, $092002(2009)$

[9] T. Happel, F. Greiner, N. Mahdizadeh, B. Nold, M. Ramisch, and U. Stroth, Phys. Rev. Lett. 102, 255001 (2009).

[10] T. Windisch, O. Grulke, V. Naulin, and T. Klinger, Plasma Phys. \& Controlled Fusion 53, 124036 (2011).

[11] J. Cheng, J. Q. Dong, L. W. Yan, K. Itoh, K. J. Zhao, W. Hong, Z. H. Hunang, L. Nie, T. Lan, A. D. Liu, et al., Nucl. Fusion 53, 093008 (2013).

[12] J. R. Myra, W. M. Davis, D. A. D'Ippolito, B. LaBombard, D. A. Russell, J. L. Terry, and S. J. Zweben, Nucl. Fusion 53, 073013 (2013).

[13] D. A. D'Ippolito, J. R. Myra, and S. J. Zweben, Phys. Plasmas 18, 060501 (2011).

[14] I. Shesterikov, Y. Xu, C. Hidalgo, M. Berte, P. Dumortier, M. Van Schnoor, M. Vergote, G. Van Oost, and the TEXTOR Team, Nucl. Fusion 52, 042004 (2012).

[15] M. Ramisch, F. Greiner, N. Mahdizadeh, K. Rahbarnia, and U. Stroth, Plasma Phys. \& Controlled Fusion 49, 777 (2007).

[16] T. A. Carter and J. E. Maggs, Phys. Plasmas 16, 012304 (2009).

[17] P. Manz, M. Ramisch, and U. Stroth, Phys. Rev. Lett. 103, 165004 (2009).

[18] M. Ramisch, P. Manz, U. Stroth, G. Birkenmeier, S. Enge, E. Holzhauer, A. Köhn, and B. Nold, Plasma Phys. \& Controlled Fusion 52, 124015 (2010).
[19] U. Stroth, P. Manz, and M. Ramisch, Plasma Phys. \& Controlled Fusion 53, 024006 (2011).

[20] B. Nold, G. D. Conway, T. Happel, H. Müller, M. Ramisch, V. Rohde, U. Stroth, and ASDEX Upgrade Team, Plasma Phys. \& Controlled Fusion 52, 065005 (2010).

[21] I. Cziegler, J. L. Terry, J. W. Hughes, and B. LaBombard, Phys. Plasmas 17, 056120 (2010).

[22] T. Happel, E. Blanco, and T. Estrada, Rev. Sci. Inst. 81, 10D901 (2010).

[23] T. Windisch, O. Grulke, V. Naulin, and T. Klinger, Plasma Phys. \& Controlled Fusion 53, 085001 (2011).

[24] G. Fuchert, G. Birkenmeier, B. Nold, M. Ramisch, and U. Stroth, Plasma Phys. \& Controlled Fusion 55, 125002 (2013).

[25] U. Stroth, F. Greiner, C. Lechte, N. Mahdizadeh, K. Rahbarnia, and M. Ramisch, Phys. Plasmas 11, 2558 (2004).

[26] B. D. Scott, Contrib. Plasma Phys. 47, 714 (2006).

[27] S. Enge, G. Birkenmeier, P. Manz, M. Ramisch, and U. Stroth, Phys. Rev. Lett. 105, 175004 (2010).

[28] A. Köhn, G. Birkenmeier, E. Holzhauer, M. Ramisch, and U. Stroth, Plasma Phys. \& Controlled Fusion 52, 035003 (2010).

[29] H. Johnsen, H. L. Pecseli, and J. Trulsen, Phys. Fluids 30, 2239 (1987).

[30] B. Nold, T. T. Ribeiro, M. Ramisch, Z. Huang, H. W. Müller, B. D. Scott, U. Stroth, and ASDEX Upgrade Team, New. J. Phys. 14, 063022 (2012).

[31] T. T. Ribeiro and B. Scott, Plasma Phys. \& Controlled Fusion 47, 1657 (2005).

[32] T. T. Ribeiro and B. Scott, Plasma Phys. \& Controlled Fusion 50, 055007 (2008).

[33] S. J. Zweben, B. D. Scott, J. L. Terry, B. LaBombard, J. W. Hughes, and D. P. Stotler, Phys. Plasmas 16, 082505 (2009)

[34] F. Gennrich and A. Kendl, Plasma Phys. \& Controlled Fusion 54, 015012 (2012).

[35] M. Kočan, F. P. Gennrich, A. Kendl, H. W. Müller, and ASDEX Upgrade Team, Plasma Phys. \& Controlled Fusion 54, 085009 (2012).

[36] J. R. Angus, M. V. Umansky, and S. I. Krasheninnikov, Phys. Rev. Lett. 108, 215002 (2012).

[37] G. S. Xu, V. Naulin, W. Fundamenski, J. J. Rasmussen, A. H. Nielsen, and B. N. Wan, Phys. Plasmas 17, 022501 (2010).

[38] N. Bisai, R. Singh, and P. K. Kaw, Phys. Plasmas 19, 052509 (2012).

[39] P. Manz, D. Carralero, G. Birkenmeier, H. W. Müller, S. H. Müller, G. Fuchert, B. D. Scott, and U. Stroth, 
Phys. Plasmas 20, 102307 (2013).

[40] J. Madsen, O. E. Garcia, J. S. Larsen, V. Naulin, A. H. Nielsen, and J. J. Rasmussen, Phys. Plasmas 18, 112504 (2011).

[41] P. Manz, G. Birkenmeier, M. Ramisch, and U. Stroth, Phys. Plasmas 19, 082318 (2012).

[42] Ö. D. Gürcan, P. H. Diamond, T. S. Hahm, and Z. Lin, Phys. Plasmas 12, 032303 (2005).

[43] O. D. Gürcan, Phys. Rev. Lett. 109, 155006 (2012).

[44] P. Ghendrih, Y. Sarazin, G. Ciraolo, G. Darmet, X. Garbet, V. Grandgirard, P. Tamain, S. Benkadda, and P. Beyer, J. Nucl. Mat. 363-365, 581 (2007).

[45] K. Lackner, R. Chodura, M. Kaufmann, J. Neuhauser,
K. G. Rauh, and W. Schneider, Plasma Phys. \& Controlled Fusion 26, 105 (1984).

[46] A. Y. Pigarov, S. I. Krasheninnikov, and B. LaBombard, Contrib. Plasma Phys. 46, 604 (2006).

[47] A. V. Chankin, D. P. Coster, G. Corrigan, S. K. Erents, W. Fundamenski, A. Kallenbach, K. Lackner, J. Neuhauser, R. Pitts, the ASDEX Upgrade Team, et al., Plasma Phys. \& Controlled Fusion 51, 065022 (2009).

[48] P. Manz, T. T. Ribeiro, B. D. Scott, G. Birkenmeier, D. Carralero, G. Fuchert, H. W. Müller, S. H. Müller, U. Stroth, and E. Wolfrum, Origin and turbulence spreading of plasma blobs to be published (2014). 